\title{
Etiology and Factors Contributing to the Severity and Mortality of Community-acquired Pneumonia
}

\author{
Takashi Ishiguro, Noboru Takayanagi, Shozaburo Yamaguchi, Hideaki Yamakawa, \\ Keitaro Nakamoto, Yotaro Takaku, Yosuke Miyahara, Naho Kagiyama, \\ Kazuyoshi Kurashima, Tsutomu Yanagisawa and Yutaka Sugita
}

\begin{abstract}
Objective Community-acquired pneumonia (CAP) remains a major cause of death. No studies have reported the use of rapid influenza diagnostic tests (RIDT) for the etiological diagnosis, and the factors contributing to severity and mortality have not yet been fully investigated. The aim of this study was to review the etiologies of CAP using RIDT and to identify risk factors related to the severity and mortality of the disease. Methods This retrospective study assessed these factors in hospitalized patients, with special emphasis on microbial etiology.

Results A total of 1,032 patients aged $63.9 \pm 18.3$ years were studied, $66.2 \%$ of whom were men. Microbial identification was obtained in $57.0 \%$ of the cases. The most frequent causative microbial agents were Streptococcus pneumoniae, Mycoplasma pneumoniae and the influenza virus, and the second most frequent pathogens in the patients with severe CAP and the non-survivors were $S$. pneumoniae and the influenza virus. Age ( $\geq 65$ years), chronic obstructive pulmonary disease, congestive heart failure, diabetes mellitus, dementia and Legionella spp. infection and polymicrobial infection were each found to be independent factors related to severity in the multivariate analysis, whereas "unidentified pathogen" was found to be an independent factor for non-severe CAP. Age ( $\geq 65$ years), chronic pulmonary aspergillosis, post-lung cancer surgery and severe CAP were found to be independent factors for non-survival according to a multivariate analysis.

Conclusion In addition to $S$. pneumoniae, the influenza virus was a frequent cause of CAP overall and a frequent causative pathogen in both severe cases of CAP and non-survivors. Legionella spp. infection and polymicrobial infection were found to be an independent factor for the severity of CAP along with advanced age and certain comorbidities. An advanced age, certain respiratory comorbidities and severe CAP were found to be important independent factors for the mortality of CAP.
\end{abstract}

Key words: community-acquired pneumonia, etiology, rapid influenza diagnostic test, severe, mortality

(Intern Med 52: 317-324, 2013)

(DOI: 10.2169/internalmedicine.52.8830)

\section{Introduction}

Despite advances in diagnosis and antimicrobial therapy, community-acquired pneumonia (CAP) remains an important cause of morbidity and mortality, especially in patients requiring hospitalization. The rates of severe $\mathrm{CAP}$ among patients hospitalized with CAP range from $6.6 \%$ to $16.7 \%$ (1). Both morbidity and mortality in patients with moderate to severe CAP remain high, and critically ill patients hospitalized with CAP have mortality rates ranging from $5 \%$ to $30-50 \%$ (2).

To improve clinically relevant outcomes, Infectious Diseases Society of America (IDSA)/American Thoracic Society (ATS) guidelines for CAP were announced in 2007 (3). Diagnostic testing to determine the etiology of CAP is encouraged, and the guidelines recommend using the rapid influenza diagnostic test (RIDT) for its epidemiologic impor- 
tance and consideration of antiviral therapy (3). To our knowledge, however, no studies reporting the routine use of RIDTs for etiological diagnosis exist. We have used RIDTs since 2002 and have administered antiviral treatment based on both the clinical diagnosis and RIDT results.

In addition, relatively few studies have reported the risk factors related to the severity and mortality of CAP. Identifying corresponding risk factors may help to define better strategies for prevention and early recognition of severe CAP and contribute to identifying patients requiring intensive care in order to mitigate the negative outcomes of CAP. The primary objective of the present study was to review the etiologies of CAP, the results of RIDTs and the outcomes of the use of neuraminidase inhibitors to treat influenza pneumonia. The secondary objective was to identify risk factors related to the severity and mortality of CAP.

\section{Materials and Methods}

We conducted a retrospective study of all patients hospitalized with CAP over a 10-year period from January 2002 through November 2011 at our institution in Saitama, Japan. CAP was diagnosed on the basis of symptoms suggestive of lower respiratory tract infections and the development of infiltrations on chest X-ray.

The excluded patients comprised those with healthcareassociated pneumonia or hospital-acquired pneumonia, as defined in the ATS/IDSA guidelines (4), those showing immunosuppression (AIDS or receiving chemotherapy), those residing in a nursing home and those with tuberculosis, nonresected lung cancer or a confirmed alternative diagnosis lasting until the end of the follow-up.

Semiquantitative culture of the microorganisms was divided into five grades: $3+, 2+, 1+$, "a little" and "very little." Microorganisms were considered causative if one of the following criteria was met: bacterial growth in sputum cultures $(\geq 2+)$, protected specimen brush samples $(\geq 1+)$, bronchoalveolar lavage fluid $(\geq 1+)$ or transbronchial aspirate $(\geq$ $1+$ ); a positive blood culture (in the absence of an apparent extrapulmonary focus); a positive bacterial culture of pleural fluid and transthoracic needle aspiration samples; or isolation of Legionella spp. or Mycoplasma pneumoniae from respiratory samples. The diagnosis of the influenza virus and atypical microorganisms, including $M$. pneumoniae, Legionella spp. and Chlamydia psittaci, was established on the basis of a 4-fold increase in the $\mathrm{IgG}$ titers of paired sera at admission and during the following three to six weeks thereafter. Increases in the antibody titers against $C$. pneumoniae (enzyme-linked immunoassay) $\geq 1.35$ for $\operatorname{IgG}$ or 1.0 for $\operatorname{IgA}$ were regarded as significant. Positive results of the urinary antigen test for $S$. pneumoniae (BinaxNOW ${ }^{\circledR}$ Streptococcus pneumoniae urinary antigen test; Binax, Inc., Portland, ME, USA), L. pneumophila (BinaxNOW ${ }^{\circledR}$ Legionella pneumophila urinary antigen test; Binax, Inc.) and RIDT for nasopharyngeal swabs (Capilia Flu ${ }^{\circledR} \mathrm{A}+\mathrm{B}$; Alfresa Pharma, Osaka, Japan [from 2002 to 2008] and ESPLINE ${ }^{\circledR}$ Influenza
A\&B-N; Fujirebio Inc., Tokyo, Japan [from 2009]) were also regarded as significant. Severe CAP was diagnosed when at least one major criterion or three minor criteria of the IDSA/ATS guidelines (3) were present.

The treatment prescribed during the first 24 hours of hospitalization was considered the initial treatment. The initial antibiotic regimen was defined as being concordant when the antibiotics chosen by the attending physician were in accordance with the recommendations of the 2007 IDSA/ATS guidelines (3), regardless of any additional administered antibiotics. The treatment effectiveness of the initial antibiotics administered after admission was judged by physicians on the basis of body temperature, physical condition and arterial oxygen saturation or partial arterial oxygen pressure.

The following variables were assessed as possible risk factors for the severity and mortality of CAP: age $(\geq 65$ years), male sex, smoking habits, presence of comorbid illnesses, history of prior antibiotics administered by a local physician and causative pathogens. The severity of CAP and the concordance of the initial antibiotic therapy with the ATS/IDSA guidelines (3) were also assessed as possible risk factors for mortality from CAP. The study protocol was approved by the Ethical Committee of the Saitama Cardiovascular and Respiratory Center.

\section{Statistical analysis}

The results are presented as the frequency and percentage or the mean $\pm \mathrm{SD}$, unless otherwise indicated. Risk factors for the severity and mortality of CAP were evaluated with univariate and multivariate logistic regression analyses. Variables showing significance in the univariate analysis $(\mathrm{p}<$ $0.05)$ were included in the multivariate logistic regression analysis with the backward elimination method. The $95 \%$ confidence intervals of all of the comparisons are also reported. In all instances, a two-tailed $\mathrm{p}$ value $<0.05$ was considered to indicate statistical significance.

\section{Results}

\section{Patient characteristics}

Overall, 1,032 patients were investigated. The characteristics of the patients diagnosed with CAP are shown in Table 1. Among these patients, 133 (12.9\%) were classified as having severe CAP and $32(3.1 \%)$ died during their hospital stay.

\section{Microbiological patterns}

The diagnostic methods used and the results obtained are listed in Table 2. Pathogens were identified in 588 (57.0\%) patients: $493(47.8 \%)$ had a single pathogen and $95(9.2 \%)$ had two or three pathogens (Table 3). The four most frequently isolated pathogens were $S$. pneumoniae, M. pneumoniae, the influenza virus and Legionella spp.

$S$. pneumoniae was the most frequent microorganism found in patients with polymicrobial infections (Table 4). 
Table 1. Patient Characteristics

\begin{tabular}{|c|c|}
\hline Characteristic & Value \\
\hline Age (years) & $63.9 \pm 18.3$ \\
\hline Male sex & $683(66.2)$ \\
\hline Pathogen identified & $588(57.0)$ \\
\hline \multicolumn{2}{|l|}{ Comorbidity } \\
\hline \multicolumn{2}{|l|}{ Pulmonary disease } \\
\hline Chronic obstructive pulmonary disease & $166(16.1)$ \\
\hline Asthma & $102(9.9)$ \\
\hline Bronchiectasis & $55(5.3)$ \\
\hline Nontuberculous mycobacteriosis & $35(3.4)$ \\
\hline Old pulmonary tuberculosis & $54(5.2)$ \\
\hline Chronic pulmonary aspergillosis & $15(1.5)$ \\
\hline Interstitial pneumonia & $43(4.2)$ \\
\hline Post lung cancer operation & $35(3.4)$ \\
\hline Other pulmonary disease & $32(3.1)$ \\
\hline \multicolumn{2}{|l|}{ Systemic disease } \\
\hline Hypertension & $114(11.0)$ \\
\hline Congestive heart failure & $35(3.4)$ \\
\hline Ischemic heart disease & $33(3.2)$ \\
\hline Diabetes mellitus & $107(10.4)$ \\
\hline Valvular heart disease & $10(1.0)$ \\
\hline Arrhythmia & $42(4.1)$ \\
\hline Cerebrovascular disease & $41(4.0)$ \\
\hline Dementia & $13(1.3)$ \\
\hline Neuromuscular disease & $10(1.0)$ \\
\hline Post upper digestive system surgery & $29(2.9)$ \\
\hline Chronic liver disease & $25(2.4)$ \\
\hline Connective tissue disease & $41(4.0)$ \\
\hline Psychological disease & $10(1.0)$ \\
\hline Malignancy & $19(1.8)$ \\
\hline Steroid or immunosuppressant & $63(6.1)$ \\
\hline Other systemic disease & $99(9.6)$ \\
\hline Severe & $133(12.9)$ \\
\hline Mortality & $32(3.1)$ \\
\hline \multicolumn{2}{|c|}{ Data presented as means \pm standard deviation or $\mathrm{n}(\%)$} \\
\hline \multicolumn{2}{|c|}{$\begin{array}{l}\text { "Other pulmonary disease" includes the following incidences of each } \\
\text { disease: pneumoconiosis }(n=7) \text {; pulmonary thromboembolism }(n=4) \text {; } \\
\text { chronic empyema }(n=4) \text {; asbestos pleuritis, sarcoidosis, pulmonary } \\
\text { alveolar proteinosis }(n=3 \text { each); and others }(n=15) \text {."Other systemic } \\
\text { disease" includes the following incidences of each disease: gastritis or } \\
\text { gastric ulcer }(n=14) \text {; mental disorder }(n=10) \text {; chronic kidney disease } \\
(n=9) \text {; chronic thyroiditis }(n=6) \text {; hyperlipidemia, postoperative breast } \\
\text { cancer, cardiomyopathy, primary immunodeficiency, alcoholism }(n=5 \\
\text { each), atrial or ventricular septal defect, prostate hypertrophy, } \\
\text { abdominal artery aneurysm }(n=4 \text { each); deep venous thrombus }(n=3) \text {; } \\
\text { and others }(n=24) \text {. Some patients have more than one commorbidity. }\end{array}$} \\
\hline
\end{tabular}

Polymicrobial infection with both $S$. pneumoniae and the influenza virus was the most frequent combination, occurring in $28(29.5 \%)$ of 95 cases. Of the 97 patients with influenza virus infection, $61(62.9 \%)$ were diagnosed according to positive RIDT and 36 (37.1\%) were diagnosed based on serology alone. Forty-one $(42.3 \%)$ of these patients were found to have polymicrobial infections. The monthly distribution of the patients diagnosed with an influenza virus infection is shown in Figure.

S. pneumoniae was the most common pathogen found in both patients with severe CAP and non-survivors, followed by the influenza virus (Table 3). Legionella spp. was the third leading cause of severe CAP and the third leading cause in the non-survivors, occurring with the same frequency as that of M. pneumoniae and H. influenzae.

\section{Risk factors for severity on admission}

A multivariate analysis found age ( $\geq 65$ years), chronic obstructive pulmonary disease (COPD), congestive heart failure $(\mathrm{CHF})$, diabetes mellitus, dementia, Legionella spp. in-
Table 2. Diagnostic Methods and Results

\begin{tabular}{lcc}
\hline Method & $\begin{array}{c}\text { No. of } \\
\text { episodes } \\
\text { studied }\end{array}$ & $\begin{array}{c}\text { No. of positive } \\
\text { diagnostic } \\
\text { studies (\%) }\end{array}$ \\
\hline Paired sera & 757 & $106(14.0)$ \\
Rapid influenza diagnostic test & 1,014 & $61(6.2)$ \\
Urinary antigen & & \\
$\quad$ Streptococcus pneumoniae & 917 & $221(24.1)$ \\
$\quad$ Legionella pneumophila & 917 & $16(1.7)$ \\
Culture & & \\
Sputum & 895 & $156(17.4)$ \\
Transbronchial aspirate & 21 & $2(9.5)$ \\
Protected specimen brush & 12 & $8(66.7)$ \\
Bronchial washing & 12 & $4(33.3)$ \\
Bronchoalveolar lavage fluid & 31 & $9(29.0)$ \\
Transthoracic aspiration & 1 & $1(100.0)$ \\
Blood & 714 & $17(2.4)$ \\
Pleural fluid & 7 & $1(14.3)$ \\
\hline
\end{tabular}

fection and polymicrobial infection to be significant factors contributing to the severity of CAP (Table 5). "Unidentified pathogen" was found to be an independent factor for nonsevere CAP. Male sex, underlying systemic disease or both systemic and respiratory disease, old pulmonary tuberculosis, cerebrovascular disease, S. pneumoniae infection and influenza virus infection were found to be significant factors according to a univariate analysis and nonsignificant factors according to a multivariate analysis.

\section{Risk factors for mortality}

The multivariate analysis showed that age ( $\geq 65$ years), chronic pulmonary aspergillosis, post-lung cancer surgery and severe CAP were independent risk factors for mortality (Table 6). Underlying systemic disease, CHF and cerebrovascular disease were each found to be significant risk factors in the univariate analysis but not in the multivariate analysis.

\section{Comparison of patients receiving guideline- concordant versus guideline-discordant therapy}

Of the 1,032 patients, $687(66.6 \%)$ received guidelineconcordant initial therapy ( $\beta$-lactams plus macrolides in 560 patients, $\beta$-lactams plus tetracyclines in 14 patients and fluoroquinolones with or without $\beta$-lactams and macrolides in 113 patients) and 345 received guideline-discordant therapy ( $\beta$-lactams only in 270 patients, macrolides only in 66 patients, tetracyclines only in three patients, $\beta$-lactams plus clindamycin in two patients, sulfamethoxazole/trimethoprim in two patients and telithromycin only in two patients). Compared with discordant therapy, concordant therapy resulted in fewer $S$. pneumoniae infections (concordant therapy: 21.8\%; discordant therapy: 27.8\%; $\mathrm{p}=0.037$ ), fewer polymicrobial infections (concordant therapy: $7.1 \%$; discordant therapy: $13.3 \% ; \mathrm{p}=0.002$ ) and less frequent treatment failure with initial antibiotics (concordant therapy: 10.9\%; discordant therapy: $15.9 \% ; \mathrm{p}=0.028$ ). There were no significant differences in other demographic variables, underlying diseases, etiology or severity between the patients who received concordant versus discordant therapy. Subsequent an- 
Table 3. Etiology of Community-acquired Pneumonia

\begin{tabular}{|c|c|c|c|c|c|c|}
\hline \multirow[b]{2}{*}{ Etiology } & \multicolumn{2}{|c|}{ Total $(n=1032)$} & \multicolumn{2}{|c|}{ Severe $(n=133)$} & \multicolumn{2}{|c|}{ Non-survivors $(\mathrm{n}=32)$} \\
\hline & No. & $(\%)$ & No. & $(\%)$ & No. & $(\%)$ \\
\hline Streptococcus pneumoniae & 246 & $(23.8)$ & 50 & $(37.6)$ & 12 & $(37.5)$ \\
\hline Mycoplasma pneumoniae & 105 & $(10.2)$ & 8 & $(6.0)$ & 2 & $(6.3)$ \\
\hline Influenza virus & 97 & $(9.4)$ & 22 & $(16.5)$ & 4 & (12.5) \\
\hline Legionella spp. & 53 & $(5.1)$ & 18 & $(13.5)$ & 2 & $(6.3)$ \\
\hline Haemophilus influenzae & 45 & (4.4) & 6 & $(4.5)$ & 2 & (6.3) \\
\hline Pseudomonas aeruginosa & 33 & (3.2) & 4 & (3.0) & 1 & (3.1) \\
\hline GNEB & 26 & $(2.5)$ & 7 & (5.3) & 1 & $(3.1)$ \\
\hline Chlamydia pneumoniae & 22 & (2.1) & 4 & $(3.0)$ & 0 & $(0)$ \\
\hline Chlamydia psittaci & 15 & (1.5) & 3 & (2.3) & 0 & $(0)$ \\
\hline Moraxella catarrhalis & 10 & $(1.0)$ & 3 & (2.3) & 1 & $(3.1)$ \\
\hline Streptococcus sp. & 9 & $(0.9)$ & 0 & (0) & 0 & $(0)$ \\
\hline Others & 29 & (2.8) & 5 & $(3.8)$ & 1 & (3.1) \\
\hline Polymicrobial infection & 95 & $(9.2)$ & 24 & $(18.0)$ & 4 & $(12.5)$ \\
\hline Unknown & 444 & $(43.0)$ & 29 & $(21.8)$ & 11 & (34.4) \\
\hline
\end{tabular}

Streptococcus sp. means other than Streptococcus pneumoniae.

GNEB: Gram-negative enteric bacilli. "Others" includes methicillin-susceptible S. aureus $(\mathrm{n}=8)$, methicillin-resistant $S$. aureus $(\mathrm{n}=3)$, Haemophilus parainfluenzae $(\mathrm{n}=2)$, Nocardia $\mathrm{sp} .(\mathrm{n}=2)$, and coagulase-negative Staphylococci, Acinetobacter baumannii, Varicella-Zoster virus, Haemophilus parahaemolyticus, Pseudomonas alcaligenes, Citrobacter freundii, Staphylococcus haemolyticus, Clostridium perfringens $(\mathrm{n}=1$ each), and anaerobes $(\mathrm{n}=6)$ (Fusobacterium sp. $[\mathrm{n}=2]$, and Prevotella sp., Gemella morbillorum, Peptostreptococcus prevotii, and Veillonella sp. $[\mathrm{n}=1 \mathrm{each}])$.

Table 4. Polymicrobial Infection

\begin{tabular}{|c|c|c|c|}
\hline Pathogens & $\begin{array}{c}\text { Total } \\
\mathrm{n}\end{array}$ & $\begin{array}{c}\text { Severe } \\
\mathrm{n}\end{array}$ & $\begin{array}{c}\text { Non-survivors } \\
\mathrm{n}\end{array}$ \\
\hline \multicolumn{4}{|l|}{2 pathogens } \\
\hline Streptococcus pneumoniae + influenza virus & 23 & 5 & 1 \\
\hline S. pneumoniae + Haemophilus influenzae & 7 & 0 & 0 \\
\hline S. pneumoniae + Chlamydophila pneumoniae & 7 & 2 & 0 \\
\hline S. pneumoniae + Mycoplasma pneumoniae & 5 & 1 & 1 \\
\hline S. pneumoniae + Legionella spp. & 4 & 3 & 0 \\
\hline S. pneumoniae + Others $^{1}$ & 9 & 4 & 0 \\
\hline Influenza virus $+M$. pneumoniae & 3 & 0 & 0 \\
\hline Influenza virus + Legionella spp. & 3 & 0 & 0 \\
\hline Influenza virus $+H$. influenzae & 3 & 1 & 1 \\
\hline Influenza virus + Others ${ }^{2}$ & 4 & 0 & 0 \\
\hline M. pneumoniae + Others $^{3}$ & 7 & 1 & 0 \\
\hline Other 2 pathogens ${ }^{4}$ & 11 & 4 & 1 \\
\hline \multicolumn{4}{|l|}{3 pathogens } \\
\hline S. pneumoniae + influenza virus + Others $^{5}$ & 5 & 2 & 0 \\
\hline 3 other pathogens ${ }^{6}$ & 4 & 1 & 0 \\
\hline \multicolumn{4}{|c|}{ 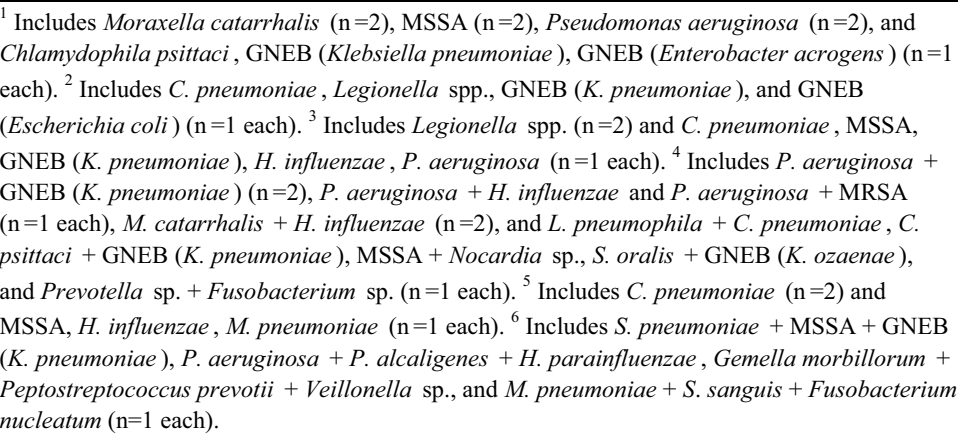 } \\
\hline
\end{tabular}

tibiotic therapy was administered in $9.2 \%$ of patients initially receiving concordant therapy and $13.0 \%$ of patients initially receiving discordant therapy.

\section{Neuraminidase inhibitors for influenza pneumonia}

Neuraminidase inhibitors were administered in 45 (46.4\%) of the 97 patients with influenza pneumonia. Administration was based on positive RIDT results in 38 pa- tients and on the clinical diagnosis only in seven patients. Four patients with influenza virus infection died; none received neuraminidase inhibitors.

\section{Discussion}

The most frequent microbial agents found in the 1,032 patients with CAP were $S$. pneumoniae, $M$. pneumoniae, the 


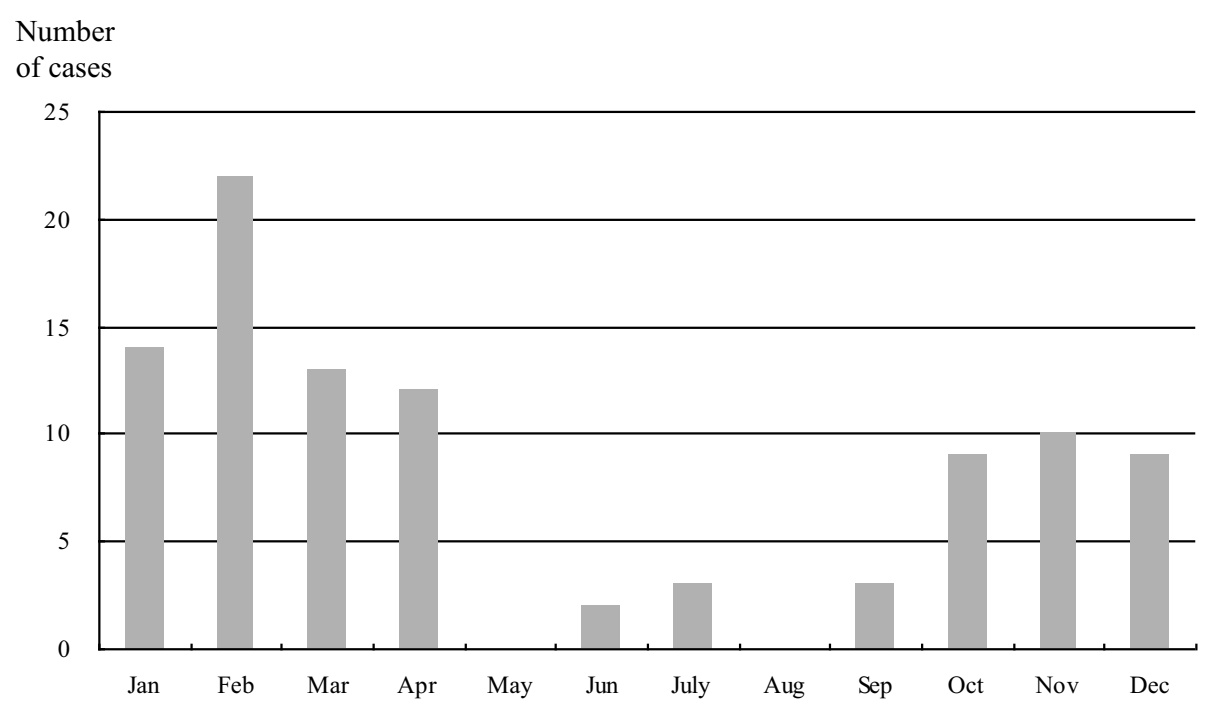

Figure. Monthly distribution of the 97 patients with influenza virus infection. The gray bars represent the number of cases of influenza virus infection diagnosed each month, with high numbers of cases diagnosed in the winter and low numbers of cases diagnosed in the summer.

influenza virus and Legionella spp. The pathogens found in the patients with severe CAP included S. pneumoniae as the most common pathogen, followed by the influenza virus, Legionella spp. and others accounting for a minor frequency. As in the patients with severe CAP, the pathogens found in the non-survivors included $S$. pneumoniae as the most common pathogen, followed by the influenza virus and $L e$ gionella spp., which occurred at equal frequency to $M$. pneumoniae and $H$. influenzae. This study, in which RIDTs were used for etiological diagnosis, elucidated that the second most frequent pathogen in patients with severe CAP and non-survivors is the influenza virus. The independent risk factors for severe CAP were age ( $\geq 65$ years), COPD, CHF, diabetes mellitus, dementia and Legionella spp. infection and polymicrobial infection. Age ( $\geq 65$ years), chronic pulmonary aspergillosis, post-lung cancer surgery and severe CAP were found to be independent risk factors for mortality, whereas guideline-concordant therapy was not.

In two previous reports of the etiologies of CAP in Japan $(5,6)$, the three most frequently isolated pathogens were S. pneumoniae, $H$. influenzae and M. pneumoniae. Diagnosis of viral infection in these studies was based on paired sera, and viruses were found in only $2.1 \%$ (5) or $3.0 \%$ (6) of patients. However, when a diagnosis of influenza virus infection is based only on paired sera, cases involving an acute clinical course or early death are missed. Although polymerase chain reaction (PCR) assays are sensitive, PCR techniques are more labor intensive, technically demanding and require specialized laboratory equipment. In contrast, RIDT is a simple test with results obtainable within 15-30 minutes. In the present study, we used this test in addition to paired sera to identify infection with influenza virus, which was found in 97 (9.4\%) of the 1,032 patients, and this result was not significantly different from that observed in Western countries $(7,8)$. Because rapid antigen tests and treatments for S. pneumoniae, the influenza virus and Legionella spp. are now available, introducing these tests into routine clinical care is encouraged, especially in cases of severe CAP.

The comparative features of influenza pneumonia can be classified into three types: primary viral pneumonia, secondary bacterial pneumonia and mixed viral and bacterial pneumonia. In the present study, $42.3 \%$ of the patients with influenza virus infection had evident polymicrobial infections and were classified as having secondary bacterial pneumonia or mixed viral and bacterial pneumonia. The other 56 cases did not always involve primary viral pneumonia because cases were included in which the pathogen causing the polymicrobial infection could not be easily identified.

Several risk factors that predict which patients will develop moderate to severe CAP, thereby requiring hospital or ICU admission, have been identified in multivariate analyses in a limited number of studies. These factors include demographics (age, sex, and residence status) and comorbid conditions (2). In our study, age ( $\geq 65$ years) and certain comorbidities were identified as being independent risk factors for severe disease and mortality. Additionally, our results also support comorbidity as an important factor for the development of severe CAP. Alcohol abuse is also a suggested risk factor for severe $\operatorname{CAP}(9,10)$; however, there were too few patients with alcohol abuse in the present study to include this variable in our analysis.

The etiology of CAP has not been identified as being a factor contributing to severity in the majority of previous studies. Although S. pneumoniae, M. pneumoniae and the influenza virus are the major pathogens of CAP, no reports have shown these microorganisms to be associated with increased severity, which is consistent with the findings of the present study. However, our results did show infection with Legionella spp. to be an independent risk factor for the de- 
Table 5. Multivariate Analysis of the Risk of Developing Severe Communityacquired Pneumonia in the Study Patients

\begin{tabular}{lccccc}
\hline Factor & $\mathrm{n}$ & Severe & OR & $95 \%$ CI & $\mathrm{p}$ value \\
\hline Age $(\geq 65$ yrs) & 578 & 100 & 2.428 & $(1.554,3.792)$ & $<0.001$ \\
Chronic obstructive pulmonary disease & 166 & 34 & 1.909 & $(1.194,3.053)$ & 0.007 \\
Congestive heart failure & 35 & 10 & 2.652 & $(1.190,5.911)$ & 0.017 \\
Diabetes mellitus & 107 & 25 & 2.441 & $(1.434,4.154)$ & 0.001 \\
Dementia & 13 & 5 & 4.230 & $(1.242,14.400)$ & 0.021 \\
Legionella spp. & 53 & 18 & 2.867 & $(1.499,5.485)$ & 0.001 \\
Number of pathogens & 493 & 79 & Reference & - & {$[<0.001]$} \\
$\quad$ Single & 95 & 24 & 1.917 & $(1.107,3.322)$ & 0.020 \\
$\quad$ Polymicrobial & 444 & 30 & 0.376 & $(0.236,0.600)$ & $<0.001$ \\
$\quad$ Unknown & & & & & \\
CI: confidence interval, OR: odds ratio & & & & & \\
Values represent p value for category against the reference. Values in brackets represent value for the \\
explanatory variable.
\end{tabular}

Table 6. Multivariate Analysis of the Risk of Mortality in the Study Patients

\begin{tabular}{lccccc}
\hline Factor & $\mathrm{n}$ & Non-survivors & OR & $95 \%$ CI & p value \\
\hline Age $(\geq 65$ yrs $)$ & 578 & 29 & 5.105 & $(1.473,17.699)$ & 0.010 \\
Chronic pulmonary aspergillosis & 15 & 2 & 6.987 & $(1.075,45.404)$ & 0.042 \\
Post lung cancer operation & 35 & 4 & 3.580 & $(1.018,12.589)$ & 0.047 \\
Severity, severe & 133 & 22 & 14.203 & $(6.426,31.393)$ & $<0.001$ \\
\hline CI: confidence interval, OR: odds ratio & & & &
\end{tabular}

velopment of severe CAP. A review of nine studies including 890 CAP patients admitted to the ICU demonstrated that Legionella spp. are common pathogens subsequent to $S$. pneumoniae (11). Another study indicated that atypical pathogens can collectively account for $\geq 20 \%$ of severe pneumonia episodes and that the dominant atypical pathogen in severe CAP is Legionella spp. (12). Similarly, Dambrava et al. assessed related etiologies according to ATS risk stratification and found Legionella spp. to be more frequent in severe cases (13). Unknown etiologies are also reported to be associated with severe CAP (13), which is contrary to our findings. It is recommended that patients with severe CAP therefore undergo several tests to identify the etiologies (3), and our use of multiple tests may have led to improved rates of pathogen identification in our patients with severe CAP.

The reported incidences of polymicrobial etiology range from $5.7 \%$ to $38.4 \%$ (7, 14-18). Polymicrobial infections accounted for $9.2 \%$ of all infections in the present study and were associated with severe CAP. S. pneumoniae was by far the most frequent copathogen in polymicrobial infections, as reported previously (14). In addition, similar to the results of this previous study, the most frequent polymicrobial pattern observed in the present study was that of S. pneumoniae and influenza virus infection. This finding underlines the importance of providing pneumococcal coverage in any initial antimicrobial treatment regimen.

Other studies also showed that the chronic comorbidities of COPD and cerebrovascular disease appear to be the most important factors for mortality in older adults hospitalized with CAP (19). Nursing home residences have also been found to be an independent risk factor for mortality (20); however, the present study included no nursing home resi- dents. To our knowledge, there are no reports suggesting the existence of associations between either chronic pulmonary aspergillosis or post-lung cancer surgery and mortality. The impaired host defenses underlying chronic pulmonary aspergillosis (21) or an impaired pulmonary function, including impaired local defense mechanisms, after lobectomy or pneumonectomy may contribute to mortality. Certainly, intercurrent disease, the most frequent of which is CAP, is the leading cause of death during postoperative follow-up care (22) for lung cancer.

Etiologies as risk factors for the mortality of CAP have not yet been sufficiently studied. In the present study, no single or polymicrobial pathogens, identified or unidentified, were found to be independent factors for mortality.

Recent studies focusing on hospitalized patients with CAP have found decreased 48-hour (23) and 30-day (24) mortality rates when initial antibiotic therapy is concordant with ATS (25) or IDSA (14) recommendations. Empirical treatment concordant with the IDSA/ATS guidelines has also been reported to improve survival in severe cases (26). In the present study, discordant therapy was not found to be an independent risk factor for mortality, although the patients receiving concordant therapy had a lower frequency of initial treatment failure than did the patients receiving discordant therapy. Our results may suggest that even when the initial treatment is not effective, patients treated with discordant therapy can receive effective subsequent treatment, resulting in a nonsignificant difference between discordant therapy and concordant therapy with respect to mortality.

Early use of neuraminidase inhibitors can reduce the development of complications such as pneumonia (27), and current CDC guidelines (28) recommend the use of neu- 
raminidase inhibitors in hospitalized patients with influenza virus infection. In the present study, 38 patients with influenza virus infection received neuraminidase inhibitors on the basis of positive RIDT results. The mortality rate for influenza pneumonia in the present study was $4.1 \%$, whereas previous studies have reported rates of 5.8-29.0\% (29-31). The efficacy of antiviral therapy and antibiotics for polymicrobial infections should thus be studied further in future studies.

The present study has several limitations. First, because this is a nonrandomized observational study, the level of confidence in the result that discordant initial treatment is not associated with mortality is reduced. Because the rate of treatment failure with initial antibiotics was higher in the discordant therapy group than in the concordant therapy group, concordant therapy may appear to be the better choice. Second, performing a complete diagnostic workup to determine the etiology was not possible in every patient. Finally, the present study was carried out in a single center, and the results may not be applicable to other settings.

In conclusion, the most frequent microbial agents of CAP are $S$. pneumoniae, $M$. pneumoniae, the influenza virus and Legionella spp. Independent risk factors for severe CAP include age ( $\geq 65$ years), COPD, CHF, diabetes mellitus, dementia and Legionella spp. infection. In addition, age $(\geq 65$ years), chronic pulmonary aspergillosis, post-lung cancer surgery and severe CAP are independent risk factors for mortality.

The authors state that they have no Conflict of Interest (COI).

\section{Acknowledgement}

We thank Hideyo Oda of Medical Toukei Co., Ltd. for his valuable advice on the statistical analysis.

\section{References}

1. Ewig S, Woodhead M, Torres A. Towards a sensible comprehension of severe community-acquired pneumonia. Intensive Care Med 37: 214-223, 2011.

2. Welte T. Risk factors and severity scores in hospitalized patients with community-acquired pneumonia: predilection of severity and mortality. Eur J Clin Microbiol Infect Dis 31: 33-47, 2012.

3. Mandell LA, Wunderink RG, Anzueto A, et al. Infectious Disease Society of America/American Thoracic Society consensus guidelines on the management of community-acquired pneumonia in adults. Clin Infect Dis 44 (Suppl 2): S27-S72, 2007.

4. American Thoracic Society; Infectious Diseases Society of America. Guidelines for the management of adults with hospitalacquired, ventilator-associated, and healthcare-associated pneumonia. Am J Respir Crit Care Med 171: 388-416, 2005.

5. Ishida T, Hashimoto T, Arita M, Ito I, Osawa M. Etiology of community-acquired pneumonia in hospitalized patients: a 3-year prospective study in Japan. Chest 114: 1588-1593, 1998.

6. Miyashita N, Fukano H, Niki Y, Matsushima T, Okimoto N. Etiology of community-acquired pneumonia requiring hospitalization in Japan. Chest 119: 1295-1296, 2000.

7. Cillóniz C, Ewig S, Polverino E, et al. Microbial aetiology of community-acquired pneumonia and its relation to severity. Thorax 66: 340-346, 2011.
8. Marcos MA, Esperatti M, Torres A. Viral pneumonia. Curr Opin Infect Dis 22: 143-147, 2009.

9. de Roux A, Cavalcanti M, Marcos MA, et al. Impact of alcohol abuse in the etiology and severity of community-acquired pneumonia. Chest 129: 1219-1225, 2006.

10. Ruiz M, Ewig S, Torres A, et al. Severe community-acquired pneumonia. Risk factors and follow-up epidemiology. Am J Respir Crit Care Med 160: 923-929, 1999.

11. Marrie TJ, Peeling RW, Fine MJ, Singer DE, Coley CM, Kapoor WN. Ambulatory patients with community-acquired pneumonia: the frequency of atypical agents and clinical course. Am J Med 101: 508-515, 1996.

12. Torres A, Serra-Batlles J, Ferrer A, et al. Severe communityacquired pneumonia. Epidemiology and prognostic factors. Am Rev Respir Dis 144: 312-318, 1991.

13. Dambrava PG, Torres A, Vallès $X$, et al. Adherence to guidelines' empirical antibiotic recommendations and community-acquired pneumonia outcome. Eur Respir J 32: 892-901, 2008.

14. Cillóniz C, Ewig S, Ferrer M, et al. Community-acquired polymicrobial pneumonia in the intensive care unit: aetiology and prognosis. Crit Care 15: R209, 2011.

15. Gutiérrez F, Masiá M, Rodriguez JC, et al. Community-acquired pneumonia of mixed etiology: prevalence, clinical characteristics, and outcome. Eur J Clin Microbiol Infect Dis 24: 377-383, 2005.

16. de Roux A, Ewig S, Garcia E, et al. Mixed community-acquired pneumonia in hospitalized patients. Eur Respir J 27: 795-800, 2006.

17. Lieberman D, Schlaeffer F, Boldur I, et al. Multiple pathogens in adult patients admitted with community-acquired pneumonia: a one year prospective study of 346 consecutive patients. Thorax 51: 179-184, 1996.

18. Lim WS, Macfarlane JT, Boswell TC, et al. Study of community acquired pneumonia aetiology (SCAPA) in adults admitted to hospital: implications for management guidelines. Thorax 56: 296301, 2001.

19. Neupane B, Walter SD, Krueger P, Marrie T, Loeb M. Predictors of inhospital mortality and re-hospitalization in older adults with community-acquired pneumonia: a prospective cohort study. BMC Geriatr 10: 22-31, 2010.

20. Kothe H, Bauer $T$, Marre $R$, et al. Outcome of communityacquired pneumonia: influence of age, residence status and antimicrobial treatment. Eur Respir J 32: 139-146, 2008.

21. Kelleher $P$, Goodsall A, Mulgirigama A, et al. Interferon- $\gamma$ therapy in two patients with progressive chronic pulmonary aspergillosis. Eur Respir J 27: 1307-1310, 2006.

22. Kim DJ, Lee JG, Lee CY, Park IK, Chung KY. Long-term survival following pneumonectomy for non-small cell lung cancer: clinical implications for follow-up care. Chest 132: 178-184, 2007.

23. Mortensen EM, Restrepo MI, Anzueto A, Pugh JA. Antibiotic therapy and 48-hour mortality for patients with pneumonia. Am J Med 119: 859-864, 2006.

24. Frei CR, Restrepo MI, Mortensen EM, Burgess DS. Impact of guideline-concordant empiric antibiotic therapy in communityacquired pneumonia. Am J Med 119: 865-871, 2006.

25. Arancibia F, Bauer TT, Ewig S, et al. Community-acquired pneumonia due to gram-negative bacteria and Pseudomonas aeruginosa: incidence, risk, and prognosis. Arch Intern Med 162: 18491858, 2002.

26. Martin-Loeches I, Lisboa T, Rodriguez A, et al. Combination antibiotic therapy with macrolides improves survival in intubated patients with community-acquired pneumonia. Intensive Care Med 36: 612-620, 2010.

27. Yu H, Liao Q, Yuan Y, et al. Effectiveness of oseltamivir on disease progression and viral RNA shedding in patients with mild pandemic 2009 influenza A H1N1: opportunistic retrospective study of medical charts in China. BMJ 341: c4779, 2010.

28. Seasonal Influenza (Flu). Centers for Disease Control and Pre- 
Intern Med 52: 317-324, 2013 DOI: 10.2169/internalmedicine.52.8830

vention. Accessed May 8, 2011, at http://www.cdc.gov/flu/ professionals/antivirals/summary-clinicians.htm

29. Oliveira EC, Marik PE, Colice G. Influenza pneumonia a descriptive study. Chest 119: 1717-1723, 2001.

30. Riquelme R, Torres A, Rioseco M, et al. Influenza pneumonia: a comparison between seasonal influenza virus and the H1N1 pandemic. Eur Respir J 38: 106-111, 2011.

31. Ruuskanen O, Lahti E, Jennings LC, Murdoch DR. Viral pneumonia. Lancet 377: 1264-1275, 2011.

(C) 2013 The Japanese Society of Internal Medicine http://www.naika.or.jp/imonline/index.html 